PROCEEDINGS OF THE

AMERICAN MATHEMATICAL SOCIETY

Volume 139, Number 10, October 2011, Pages 3601-3610

S 0002-9939(2011)10981-X

Article electronically published on March 9, 2011

\title{
POWER MEANS \\ GENERATED BY SOME MEAN-VALUE THEOREMS
}

\author{
JANUSZ MATKOWSKI
}

(Communicated by Edward C. Waymire)

\begin{abstract}
According to a new mean-value theorem, under the conditions of a function $f$ ensuring the existence and uniqueness of Lagrange's mean, there exists a unique mean $M$ such that

$$
\frac{f(x)-f(y)}{x-y}=M\left(f^{\prime}(x), f^{\prime}(y)\right) .
$$

The main result says that, in this equality, $M$ is a power mean if, and only if, $M$ is either geometric, arithmetic or harmonic. A Cauchy relevant type result is also presented.
\end{abstract}

\section{INTRODUCTION}

In a recent paper [4] the following counterpart of the Lagrange mean-value theorem has been proved. If a real function $f$ defined on an interval $I \subset \mathbb{R}$ is differentiable, and $f^{\prime}$ is one-to-one, then there exists a unique mean function $M: f^{\prime}(I) \times f^{\prime}(I) \rightarrow f^{\prime}(I)$, such that

$$
\frac{f(x)-f(y)}{x-y}=M\left(f^{\prime}(x), f^{\prime}(y)\right), \quad x, y \in I, x \neq y .
$$

In this connection the following problem arises. Given a mean $M$, determine all differentiable real functions $f$ such that

$$
\frac{f(x)-f(y)}{x-y}=M\left(f^{\prime}(x), f^{\prime}(y)\right), \quad x, y \in I, x \neq y .
$$

In the case when $M$ is the geometric mean this equation has appeared in 3 and was useful in solving an open problem related to convex functions (cf. Remark 5).

In the first section we consider equation (1) in the case when $M=M^{[\varphi]}$, where

$$
M^{[\varphi]}(u, v)=\varphi^{-1}\left(\frac{\varphi(u)+\varphi(v)}{2}\right), \quad u, v \in J,
$$

and $\varphi: f^{\prime}(I) \rightarrow \mathbb{R}$ is a continuous and strictly monotonic function; so $M$ is a quasiarithmetic mean of a generator $\varphi$. Assuming three times continuous differentiability of $f$, and twice continuous differentiability of $\varphi$, we give some necessary conditions for equality (1) (Theorem 1). Applying this result, in the next section we give a

Received by the editors August 26, 2010.

2010 Mathematics Subject Classification. Primary 26A24, 26E60; Secondary 39 B22.

Key words and phrases. Mean-value theorem, mean, quasi-arithmetic mean, arithmetic mean, geometric mean, harmonic mean, Jensen functional equation, differential equation. 
complete solution of the problem in the case when $M^{[\varphi]}$ is positively homogeneous, that is, when either $\varphi(t)=A t^{p}+B$ or $\varphi(t)=A \log t+B$ for some real $p, A, B$ such that $A \neq 0 \neq p$. Then $M=M^{[p]}:(0, \infty)^{2} \rightarrow(0, \infty)$ is a power mean, that is,

$$
M^{[p]}(u, v):=\left\{\begin{array}{cl}
\left(\frac{u^{p}+v^{p}}{2}\right)^{1 / p} & \text { if } p \neq 0, \\
\sqrt{u v} & \text { if } p=0 .
\end{array}\right.
$$

The main result (Theorem 2) says that equality (1) with $M=M^{[p]}$ holds if, and only if, the mean $M$ is either arithmetic $\left(M^{[1]}(u, v)=\frac{u+v}{2}\right)$, geometric $\left(M^{[0]}(u, v)=\right.$ $\sqrt{u v})$ or harmonic $\left(M^{[-1]}(u, v)=\frac{2 u v}{u+v}\right)$.

Assume that the functions $f, g: I \rightarrow \mathbb{R}$ satisfy the conditions ensuring the existence and uniqueness of the classical Cauchy mean-value. Then (cf. 4]) there exists a unique mean $M: J^{2} \rightarrow J$, with $J:={\frac{f^{\prime}}{g^{\prime}}}^{\prime}(I)$, such that

$$
\frac{f(x)-f(y)}{g(x)-g(y)}=M\left(\frac{f^{\prime}(x)}{g^{\prime}(x)}, \frac{f^{\prime}(y)}{g^{\prime}(y)}\right), \quad x, y \in I, x \neq y .
$$

Applying Theorem 2, we determine all power means $M$ and the functions $f, g$ satisfying this equation.

\section{The CASE When $M$ IS QUASI-ARITHMETIC}

In this section we prove:

Theorem 1. Let $I, J \subset \mathbb{R}$ be intervals. Suppose that

$f: I \rightarrow \mathbb{R}$ is three times continuously differentiable, $f^{\prime \prime}(x) \neq 0$ for $x \in I$;

$\varphi: J \rightarrow \mathbb{R}$ is twice continuously differentiable, and $\varphi^{\prime}(u) \neq 0$ for $u \in J$. If

$$
\frac{f(x)-f(y)}{x-y}=M^{[\varphi]}\left(f^{\prime}(x), f^{\prime}(y)\right), \quad x, y \in I, x \neq y,
$$

then there exists $C \in \mathbb{R}, C \neq 0$, such that

$$
\varphi^{\prime}\left(f^{\prime}(x)\right)=\frac{C}{f^{\prime \prime}(x)^{2 / 3}}, \quad x \in I,
$$

and

(4)

$f^{\prime \prime}(x)\left(f^{\prime}(x)-\frac{f(x)-f(y)}{x-y}\right)^{3}=f^{\prime \prime}(y)\left(\frac{f(x)-f(y)}{x-y}-f^{\prime}(y)\right)^{3}, \quad x, y \in I, x \neq y$.

Proof. Without any loss of generality we can assume that $\varphi^{\prime}(x)>0$ in $J$. Suppose that (2) holds true. Then, from the definition of the quasi-arithmetic mean,

$$
2 \varphi\left(\frac{f(x)-f(y)}{x-y}\right)=\varphi\left(f^{\prime}(x)\right)+\varphi\left(f^{\prime}(y)\right), \quad x, y \in I, x \neq y .
$$

Differentiating both sides, first with respect to $x$ and then with respect to $y$, we get

$$
\begin{aligned}
& 2 \varphi^{\prime}\left(\frac{f(x)-f(y)}{x-y}\right) \frac{f^{\prime}(x)(x-y)-f(x)+f(y)}{(x-y)^{2}}=\varphi^{\prime}\left(f^{\prime}(x)\right) f^{\prime \prime}(x), \\
& 2 \varphi^{\prime}\left(\frac{f(x)-f(y)}{x-y}\right) \frac{f^{\prime}(y)(y-x)-f(y)+f(x)}{(x-y)^{2}}=\varphi^{\prime}\left(f^{\prime}(y)\right) f^{\prime \prime}(y)
\end{aligned}
$$


for all $x, y \in I, x \neq y$. Subtracting the respective sides of these equalities and dividing the obtained differences by $x-y$ we have

$$
\begin{aligned}
& 2 \varphi^{\prime}\left(\frac{f(x)-f(y)}{x-y}\right) \frac{\left[f^{\prime}(x)+f^{\prime}(y)\right](x-y)-2[f(x)-f(y)]}{(x-y)^{3}} \\
& =\frac{\varphi^{\prime}\left(f^{\prime}(x)\right) f^{\prime \prime}(x)-\varphi^{\prime}\left(f^{\prime}(y)\right) f^{\prime \prime}(y)}{x-y}
\end{aligned}
$$

for all $x, y \in I, x \neq y$. Applying L'Hospital's rule (or Taylor's theorem) we easily get

$$
\lim _{y \rightarrow x} \frac{\left[f^{\prime}(x)+f^{\prime}(y)\right](x-y)-2[f(x)-f(y)]}{(x-y)^{3}}=\frac{f^{\prime \prime \prime}(x)}{6}, \quad x \in I .
$$

Hence, letting $y \rightarrow x$ in (7), we obtain

$$
2 \varphi^{\prime}\left(f^{\prime}(x)\right) \frac{f^{\prime \prime \prime}(x)}{6}=\varphi^{\prime \prime}\left(f^{\prime}(x)\right)\left[f^{\prime \prime}(x)\right]^{2}+\varphi^{\prime}\left(f^{\prime}(x)\right) f^{\prime \prime \prime}(x),
$$

whence

$$
3 \varphi^{\prime \prime}\left(f^{\prime}(x)\right)\left[f^{\prime \prime}(x)\right]^{2}+2 \varphi^{\prime}\left(f^{\prime}(x)\right) f^{\prime \prime \prime}(x)=0, \quad x \in I .
$$

Assume first that $f^{\prime \prime}(x) \neq 0$ for all $x \in I$. Then, by the Darboux property of a derivative, $f^{\prime \prime}$ is of a constant sign in $I$. Dividing both sides by $f^{\prime \prime}(x) \varphi^{\prime}\left(f^{\prime}(x)\right)$ we hence get

or, equivalently,

$$
3 \frac{\varphi^{\prime \prime}\left(f^{\prime}(x)\right)}{\varphi^{\prime}\left(f^{\prime}(x)\right)} f^{\prime \prime}(x)+2 \frac{f^{\prime \prime \prime}(x)}{f^{\prime \prime}(x)}=0, \quad x \in I,
$$

$$
\left(3 \log \varphi^{\prime}\left(f^{\prime}(x)\right)+2 \log f^{\prime \prime}(x)\right)^{\prime}=0, \quad x \in I,
$$

whence, after simple calculation,

$$
\varphi^{\prime}\left(f^{\prime}(x)\right)=\frac{C}{f^{\prime \prime}(x)^{2 / 3}}, \quad x \in I
$$

for some $C \in \mathbb{R}, C \neq 0$.

Hence, dividing the respective sides of (5) and (6) (by the assumption it can be done), we obtain

$$
\frac{f^{\prime}(x)(x-y)-f(x)+f(y)}{f^{\prime}(y)(y-x)-f(y)+f(x)}=\left(\frac{f^{\prime \prime}(y)}{f^{\prime \prime}(x)}\right)^{1 / 3}, \quad x, y \in I, x \neq y,
$$

which implies (4). This completes the proof.

Remark 1. The function $f(x)=\alpha x+\beta, x \in I$, satisfies equation (1) with an arbitrary mean $M: J^{2} \rightarrow J$. Therefore in Theorem 1 we assume that $f^{\prime}$ is not a constant function.

Remark 2. From equation (1) we have

$$
f(x)-f(y)=M\left(f^{\prime}(x), f^{\prime}(y)\right)(x-y), \quad x, y \in I .
$$

Since $f(x)-f(y)=[f(x)-f(z)]+[f(z)-f(y)]$, for all $x, y, z \in I$, we get

$$
M\left(f^{\prime}(x), f^{\prime}(y)\right)(x-y)=M\left(f^{\prime}(x), f^{\prime}(z)\right)(x-z)+M\left(f^{\prime}(z), f^{\prime}(y)\right)(z-y) .
$$

Assuming that $f^{\prime}$ is one-to-one and putting $h:=\left(f^{\prime}\right)^{-1}$, we hence obtain

$$
M(u, v)[h(u)-h(v)]=M(u, w)[h(u)-h(w)]+M(w, v)[h(w)-h(v)]
$$

for all $u, v, w \in J:=f^{\prime}(I)$. 
If $f(x)=\frac{a}{2} x^{2}+b x+c$ for some $a, b, c \in \mathbb{R}$, then $h(u)=\frac{u-b}{a}$. It is easy to verify that $h$ and $M(u, v)=\frac{u+v}{2}$ satisfy this equality.

\section{The CASE WHEN $M$ IS A POWER MEAN}

In this part we assume that $M$ in equation (1) is a power mean. The main result reads as follows.

Theorem 2. Let $I \subset \mathbb{R}$ be an interval. Suppose that $f: I \rightarrow \mathbb{R}$ is differentiable, $f^{\prime}(x)>0$ for $x \in I$, and $f^{\prime}$ is not constant in $I$. Then

$$
\frac{f(x)-f(y)}{x-y}=M^{[p]}\left(f^{\prime}(x), f^{\prime}(y)\right), \quad x, y \in I, x \neq y,
$$

for some $p \in \mathbb{R}$ if, and only if, one of the following cases occurs:

(i): $p=0$ (that is, $M^{[p]}$ is the geometric mean) and for some $a, b, c \in \mathbb{R}$, $a c-b \neq 0$,

$$
f(x)=\frac{a x+b}{x+c}, \quad x \in I
$$

(ii): $p=1$ (that is, $M^{[p]}$ is the arithmetic mean) and for some $a, b, c \in \mathbb{R}$, $a \neq 0$,

$$
f(x)=\frac{a}{2} x^{2}+b x+c, \quad x \in I ;
$$

(iii): $p=-1$ (that is, $M^{[p]}$ is the harmonic mean) and for some $a, b, c, k \in \mathbb{R}$, $a \neq 0 \neq k$,

$$
f(x)=k \sqrt{a x+b}+c, \quad x \in I .
$$

Proof. First consider the case when $p=0$. If $f$ satisfies equation (8), then

$$
\frac{f(x)-f(y)}{x-y}=\sqrt{f^{\prime}(x) f^{\prime}(y)}, \quad x, y \in I, x \neq y,
$$

whence

$$
f(x)-f(y)=\sqrt{f^{\prime}(x) f^{\prime}(y)}(x-y), \quad x, y \in I .
$$

Since $f(x)-f(y)=[f(x)-f(z)]+[f(z)-f(y)]$ for all $x, y, z \in I$, we hence get

$$
\sqrt{f^{\prime}(x) f^{\prime}(y)}(x-y)=\sqrt{f^{\prime}(x) f^{\prime}(z)}(x-z)+\sqrt{f^{\prime}(z) f^{\prime}(y)}(z-y), \quad x, y, z \in I .
$$

Setting here $z=\frac{x+y}{2}, x \neq y$, and then dividing both sides by $x-y$, we get

$$
2 \sqrt{f^{\prime}(x) f^{\prime}(y)}=\sqrt{f^{\prime}(x) f^{\prime}\left(\frac{x+y}{2}\right)}+\sqrt{f^{\prime}\left(\frac{x+y}{2}\right) f^{\prime}(y)},
$$

which, obviously, also remains true for all $x, y \in I$. Dividing both sides of this equality by $2 \sqrt{f^{\prime}(x) f^{\prime}\left(\frac{x+y}{2}\right) f^{\prime}(y)}$ we hence get

$$
\frac{1}{\sqrt{f^{\prime}\left(\frac{x+y}{2}\right)}}=\frac{\frac{1}{\sqrt{f^{\prime}(x)}}+\frac{1}{\sqrt{f^{\prime}(y)}}}{2}, \quad x, y \in I
$$


that is, $\frac{1}{\sqrt{f^{\prime}\left(\frac{x+y}{2}\right)}}$ is the arithmetic mean of $\frac{1}{\sqrt{f^{\prime}(x)}}$ and $\frac{1}{\sqrt{f^{\prime}(y)}}$ (and $\sqrt{f^{\prime}\left(\frac{x+y}{2}\right)}$ is the harmonic mean of $\sqrt{f^{\prime}(x)}$ and $\sqrt{f^{\prime}(y)}$ ). It follows that the function $\gamma:=1 / \sqrt{f^{\prime}}$ satisfies the Jensen functional equation

$$
\gamma\left(\frac{x+y}{2}\right)=\frac{\gamma(x)+\gamma(y)}{2}, \quad x, y \in I .
$$

Since $\gamma$ is Lebesgue measurable, there are $k, m \in \mathbb{R}$ such that $\gamma(x)=k x+m$, for all $x \in I$ (cf. M. Kuczma [2, Chapter XIII, Section 2]. Hence

$$
f^{\prime}(x)=\frac{1}{(k x+m)^{2}}, \quad x \in I,
$$

where $k \neq 0$, as $f^{\prime}$ is not constant, whence, for some real $a$,

$$
f(x)=a-\frac{1}{k(k x+m)}=\frac{a x+\left(\frac{a m}{k}-\frac{1}{k^{2}}\right)}{x+\frac{m}{k}}, \quad x \in I .
$$

Setting $b:=\frac{a m}{k}-\frac{1}{k^{2}}, c:=\frac{m}{k}$ we get

$$
f(x)=\frac{a x+b}{x+c}, \quad x \in I,
$$

and

$$
a c-b=a \frac{m}{k}-\left(\frac{a m}{k}-\frac{1}{k^{2}}\right)=\frac{1}{k^{2}} \neq 0 .
$$

It is easy to verify that $f$ satisfies equation (8).

Now assume that $p \neq 0$. In this case,

$$
\varphi(t)=A t^{p}+B, \quad t>0,
$$

for some $A, B \in \mathbb{R}, A \neq 0$, is a generator of the mean $M^{[p]}$, and equation (8) can be written in the form

$$
\frac{f(x)-f(y)}{x-y}=\left(\frac{\left[f^{\prime}(x)\right]^{p}+\left[f^{\prime}(y)\right]^{p}}{2}\right)^{1 / p}, \quad x, y \in I, x \neq y .
$$

Assume that $f: I \rightarrow \mathbb{R}$ satisfies this equation. Then, obviously, $f$ is of the class $C^{\infty}$ in $I$.

Let $I_{0} \subset I$ be a maximal open and non-empty interval such that $f^{\prime \prime}(x) \neq 0$ for all $x \in I_{0}$. By the Darboux property of a derivative, $f^{\prime \prime}$ is of a constant sign in $I_{0}$. Since, by assumption, $f^{\prime \prime}$ does not vanish everywhere in $I$, such an interval exists. In view of Theorem 1 , there is $C \neq 0$ such that

$$
p A\left[f^{\prime}(x)\right]^{p-1}=\frac{C}{\left|f^{\prime \prime}(x)\right|^{2 / 3}}, \quad x \in I_{0},
$$

whence

$$
\left[f^{\prime}(x)\right]^{p-1}\left|f^{\prime \prime}(x)\right|^{2 / 3}=\frac{C}{p A}, \quad x \in I_{0} .
$$

If $p=1$ we hence get

$$
f^{\prime \prime}(x)=a, \quad x \in I_{0},
$$

for some $a \in \mathbb{R}, \quad a \neq 0$. Consequently

$$
f(x)=\frac{a}{2} x^{2}+b x+c, \quad x \in I_{0},
$$


for some $b, c \in \mathbb{R}$ and, obviously, $I=I_{0}$. Since

$$
\frac{f(x)-f(y)}{x-y}=a \frac{x+y}{2}+b=\frac{(a x+b)+(a y+b)}{2}=M^{[1]}\left(f^{\prime}(x), f^{\prime}(y)\right)
$$

for all $x, y \in I, x \neq y$, equation (8) is satisfied.

In the sequel we assume that $p \neq 1$. Since $f^{\prime \prime}$ is of a constant sign in $I_{0}$, from (9) we get

$$
\left[f^{\prime}(x)\right]^{q} f^{\prime \prime}(x)=a, \quad x \in I_{0},
$$

for some $a \neq 0$ and

$$
q:=\frac{3}{2}(p-1) .
$$

If $q=-1$, then (10) implies that

$$
\log f^{\prime}(x)=a x+b, \quad x \in I_{0},
$$

for some $b \in \mathbb{R}$, whence

$$
f(x)=\frac{1}{a} e^{a x+b}+\delta, \quad x \in I_{0},
$$

and, of course, $I=I_{0}$. From (11) we get

$$
p=\frac{1}{3} .
$$

Since

$$
\frac{f(x)-f(y)}{x-y}=\frac{e^{a x+b}-e^{a y+b}}{a(x-y)}, \quad x, y \in I, x \neq y,
$$

and

$$
M^{[1 / 3]}\left(f^{\prime}(x), f^{\prime}(y)\right)=\left(\frac{\left[e^{a x+b}\right]^{1 / 3}+\left[e^{a y+b}\right]^{1 / 3}}{2}\right)^{3}, \quad x, y \in I, x \neq y,
$$

equation (8) is not fulfilled.

If $q \neq-1$, then, from (10),

$$
\frac{1}{q+1}\left[f^{\prime}(x)\right]^{q+1}=a x+b, \quad x \in I_{0} ;
$$

that is,

$$
f^{\prime}(x)=[(q+1)(a x+b)]^{1 /(q+1)}, \quad x \in I_{0} .
$$

For $q=-2$, we hence get

$$
f^{\prime}(x)=-\frac{1}{a x+b}, \quad x \in I_{0},
$$

whence, for some $m \in \mathbb{R}$,

$$
f(x)=-\log |a x+b|+m, \quad x \in I_{0},
$$

and, of course, $I=I_{0}$. In this case, (11) implies that

$$
p=-\frac{1}{3} \text {. }
$$


From (13) we have

$$
\begin{gathered}
\frac{f(x)-f(y)}{x-y}=\frac{1}{x-y} \log \left|\frac{a y+b}{a x+b}\right|, \quad x, y \in I, \quad x \neq y, \\
M^{[-1 / 3]}\left(f^{\prime}(x), f^{\prime}(y)\right)=\frac{8}{(\sqrt[3]{a x+b}+\sqrt[3]{a y+b})^{3}}, \quad x, y \in I,
\end{gathered}
$$

and, obviously, equation (8) is not satisfied.

Assume that $q \notin\{-1,-2\}$. From (12) we get

$$
f(x)=\frac{1}{(q+2) a}[(q+1)(a x+b)]^{(q+2) /(q+1)}+m, \quad x \in I_{0},
$$

for some $k, m, r \in \mathbb{R}, k \neq 0$. Thus

$$
f(x)=k(a x+b)^{r}+m, \quad x \in I_{0},
$$

where

$$
r:=\frac{q+2}{q+1} \neq 0, \quad k:=\frac{(q+1)^{(q+2) /(q+1)}}{(q+2) a} \neq 0 .
$$

Making use of (14) and setting $u:=a x+b, v:=a y+b$ for $x, y \in I_{0}, x \neq y$, we get

$$
\frac{f(x)-f(y)}{x-y}=k \frac{(a x+b)^{r}-(a y+b)^{r}}{x-y}=k \frac{u^{r}-v^{r}}{\frac{u-b}{a}-\frac{v-b}{a}}=k a \frac{u^{r}-v^{r}}{u-v},
$$

and

$$
f^{\prime}(x)=\operatorname{kar}(a x+b)^{r-1}, f^{\prime}(y)=\operatorname{kar}(a y+b)^{r-1}
$$

Thus

$$
\begin{aligned}
M^{[p]}\left(f^{\prime}(x), f^{\prime}(y)\right) & =\operatorname{kar}\left(\frac{(a x+b)^{p(r-1)}+(a y+b)^{p(r-1)}}{2}\right)^{1 / p} \\
& =\operatorname{kar}\left(\frac{u^{p(r-1)}+v^{p(r-1)}}{2}\right)^{1 / p}
\end{aligned}
$$

whence, by (8),

$$
\frac{u^{r}-v^{r}}{u-v}=r\left(\frac{u^{p(r-1)}+v^{p(r-1)}}{2}\right)^{1 / p}
$$

for all $u, v \in J_{0}:=a I_{0}+b, u \neq v$. The right side,

$$
R(u, v):=r\left(\frac{u^{p(r-1)}+v^{p(r-1)}}{2}\right)^{1 / p},
$$

is an analytic function (in the real sense) in $(0, \infty)^{2}$. This equality implies that the function on the left side extended to the diagonal by the formula

$$
L(u, v):= \begin{cases}\frac{u^{r}-v^{r}}{u-v} & \text { for } u \neq v, \\ r u^{r-1} & \text { for } u=v\end{cases}
$$

is also analytic in $(0, \infty)^{2}$ (cf. also Remark 3 below) and we have

$$
R(u, v)-L(u, v)=0, \quad u, v>0 .
$$


Setting $v=1$ we get

$$
g(u):=R(u, 1)-L(u, 1)=0, \quad u>0,
$$

and, of course,

$$
g^{(k)}(1)=0 \text { for all } k \in\{0,1, \ldots\} .
$$

After some calculations we get

$$
g^{\prime \prime}(1)=\frac{1}{12} r(1-r)[3 p(r-1)-(r+1)]
$$

and

$$
\begin{aligned}
g^{(4)}(1)= & \frac{1}{80} r(r-1)\left[10 p^{3}(r-1)^{3}+15 p^{2}(1-r)^{3}+10 p(1-r)\left(3 r^{2}-24 r+43\right)\right. \\
& \left.+11 r^{3}-69 r^{2}+61 r+141\right] .
\end{aligned}
$$

Since $p \neq 0$ and $p \neq 1$, from the equality $g^{\prime \prime}(1)=0$ we get

$$
p=\frac{r+1}{3(r-1)} \text {. }
$$

Setting this value into the equality $g^{(4)}(1)=0$ we get

$$
g^{(4)}(1)=\frac{r(2-r)(r-1)(r+1)(2 r-1)}{540}=0 .
$$

By (15) and (16), respectively, we can omit the cases when $r=0$ or $r=1$. If $r=-1$, then, by (16), we get $p=0$, the case already considered. Therefore, applying (16), we conclude that either $r=2$ and $p=1$ or $r=\frac{1}{2}$ and $p=-1$.

If $r=2$ and $p=1$, then, by (14), we get

$$
f(x)=k(a x+b)^{2} \text { for } x \in I_{0} \quad \text { and } \quad M^{[1]}(u, v)=\frac{u+v}{2}, \quad u, v>0,
$$

and, if $r=\frac{1}{2}$ and $p=-1$, we get

$$
f(x)=k \sqrt{a x+b} \text { for } x \in I_{0} \quad \text { and } \quad M^{[-1]}(u, v)=\frac{2 u v}{u+v}, \quad u, v>0 .
$$

It is easy to verify that in both cases equation (8) is fulfilled. Moreover, in each of these cases, the regularity of the solutions implies that $I=I_{0}$. This completes the proof.

Remark 3 . The analyticity of the function $L$ can also be obtained as follows. Treating $L$ as a function of two complex variables, $u$ and $v$, it is easy to see that, at any point of the diagonal points $(u, u) \neq(0,0)$, the function $L$ is separately analytic (holomorphic) at $(u, u)$ with respect to each variable. Therefore, by the famous theorem of Hartogs [1], $L$ is analytic at $(u, u)$ with respect to both variables.

Remark 4. The necessity of the positivity of $f^{\prime}$ in Theorem 2 follows from the definition of the power means. Defining $M^{[p]}:(-\infty, 0)^{2} \rightarrow(-\infty, 0)$ by the formula

$$
M^{[p]}(u, v):=\left\{\begin{array}{ccc}
-\left(\frac{(-u)^{p}+(-v)^{p}}{2}\right)^{1 / p} & \text { if } & p \neq 0, \\
-\sqrt{u v} & \text { if } & p=0,
\end{array}\right.
$$

we can formulate the counterpart of Theorem 2 for $f$ such that $f^{\prime}<0$. 
Remark 5. Equation (8) for $p=0$ has appeared to be useful in solving a problem of Zs. Páles concerning existence of discontinuous Jensen affine (convex and concave) functions in the sense of Beckenbach with respect to the two-parameter family of curves generated by a Tchebycheff system [3].

Applying Theorem 2 we can prove the following:

Theorem 3. Let $I \subset \mathbb{R}$ be an interval. Suppose that $f, g: I \rightarrow \mathbb{R}$ are differentiable, $f^{\prime}(x) g^{\prime}(x)>0$ for $x \in I$ and $f^{\prime} / g^{\prime}$ is not constant in $I$. If $M$ is a power mean, then

$$
\frac{f(x)-f(y)}{g(x)-g(y)}=M\left(\frac{f^{\prime}(x)}{g^{\prime}(x)}, \frac{f^{\prime}(y)}{g^{\prime}(y)}\right), \quad x, y \in I, x \neq y,
$$

if, and only if, one of the following cases occurs:

(i): $M$ is the geometric mean, $g$ is arbitrary, and for some $a, b, c \in \mathbb{R}, a c-b \neq$ 0 ,

$$
f(x)=\frac{a g(x)+b}{g(x)+c}, \quad x \in I ;
$$

(ii): $M$ is the arithmetic mean, $g$ is arbitrary, and for some $a, b, c \in \mathbb{R}, a \neq 0$,

$$
f(x)=\frac{a}{2} g(x)^{2}+b g(x)+c, \quad x \in I ;
$$

(iii): $M$ is the harmonic mean, $g$ is arbitrary, and for some $a, b, c, k \in \mathbb{R}$, $a \neq 0 \neq k$,

$$
f(x)=k \sqrt{a g(x)+b}+c, \quad x \in I .
$$

Proof. Suppose that the functions $f$ and $g$ satisfy the assumptions and equation (17) where $M=M^{[p]}$ for some $p \in \mathbb{R}$. Obviously $g(I)$ is an interval and the function $f \circ g^{-1}: g(I) \rightarrow \mathbb{R}$ satisfies all assumptions of Theorem 2. Take arbitrary $u, v \in g(I), u \neq v$. Setting $x:=g^{-1}(u), y:=g^{-1}(v)$ in (17) we get

$$
\begin{aligned}
\frac{f \circ g^{-1}(u)-f \circ g^{-1}(v)}{u-v} & =M^{[p]}\left(\frac{f^{\prime} \circ g^{-1}(u)}{g^{\prime} \circ g^{-1}(u)}, \frac{f^{\prime} \circ g^{-1}(v)}{g^{\prime} \circ g^{-1}(v)}\right) \\
& =M^{[p]}\left(\left(f \circ g^{-1}\right)^{\prime}(u),\left(f \circ g^{-1}\right)^{\prime}(v)\right),
\end{aligned}
$$

which means that the function $f \circ g^{-1}$ satisfies equation (8). Theorem 2 implies that either $p=0$ and for some $a, b, c \in \mathbb{R}, a c-b \neq 0$,

$$
f \circ g^{-1}(u)=\frac{a u+b}{u+c}, \quad u \in g(I)
$$

or $p=1$ and for some $a, b, c \in \mathbb{R}, a \neq 0$,

$$
f \circ g^{-1}(u)=\frac{a}{2} u^{2}+b u+c, \quad u \in g(I),
$$

or $p=-1$ and for some $a, b, c, k \in \mathbb{R}, a \neq 0 \neq k$,

$$
f \circ g^{-1}(u)=k \sqrt{a u+b}+c, \quad u \in g(I) .
$$

Setting in these formulas $u=g(x)$ for $x \in I$, we obtain the result. 


\section{FinAL REMARK}

Replacing $f^{\prime}$ by $h$ of the equation for $p=0, p=1$, and $p=-1$ (the cases itemized in Theorem 2) we get three functional equations:

$$
\begin{array}{ll}
\frac{f(x)-f(y)}{x-y}=\sqrt{h(x) h(y)}, & x, y \in I, x \neq y, \\
\frac{f(x)-f(y)}{x-y}=\frac{h(x)+h(y)}{2}, & x, y \in I, x \neq y, \\
\frac{f(x)-f(y)}{x-y}=\frac{2 h(x) h(y)}{h(x)+h(y)}, & x, y \in I, x \neq y .
\end{array}
$$

It is not difficult to show that, without any regularity assumptions on $f$ and $h$, each of these equations characterizes the respective function $f$ and its derivative $h$.

It is interesting that this fact remains true if we "pexiderize" these equations by replacing $f(y)$ by $\phi(y)$ and $h(y)$ by $\gamma(y)$.

\section{REFERENCES}

1. F. Hartogs, Zur Theorie der analytischen Funktionen mehrerer unabhängiger Veränderlichen, Math. Ann. 62 (1906), 1-88. MR.1511365

2. M. Kuczma, An Introduction to the Theory of Functional Equations and Inequalities. Cauchy's Equation and Jensen's Inequality, Uniwersytet Śläski - PWN, Warszawa - Kraków - Katowice, 1985 (Second edition, edited with a preface of Attila Gilányi, Birkhäuser Verlag, Basel, 2009). MR 788497 (86i:39008)

3. J. Matkowski, Generalized convex functions and a solution of a problem of Zs. Páles, Publ. Math. Debrecen, 73/3-4 (2008), 421-460. MR2466385 (2009i:26048)

4. J. Matkowski, A mean-value theorem and its applications, J. Math. Anal. Appl. 373 (2011), 227-234. MR 2684472

Faculty of Mathematics, Computer Science and Econometrics, University of Zielona Góra, Podgórna 50, PL-65246 Zielona Góra, Poland - and - Institute of Mathematics, Silesian University, Bankowa 14, PL-42007 Katowice, Poland

E-mail address: J.Matkowski@wmie.uz.zgora.pl 